\title{
Perfil lipídico e peroxidação de lipídeos no plasma em crianças e adolescentes com sobrepeso e obesidade
}

\author{
Plasma lipid profile and lipid peroxidation in overweight or obese children and adolescents \\ Severina C.V.C. Lima1, Ricardo F. Arrais², Maria G. Almeida ${ }^{3}$, Zélia M. Souza4, Lúcia F.C. Pedrosa5
}

\section{Resumo}

Objetivo: Identificar o perfil lipídico e a peroxidação de lipídeos no plasma de crianças e adolescentes com sobrepeso e obesidade atendidos no Ambulatório de Endocrinologia Pediátrica do HOSPED/UFRN.

Métodos: Foram constituídos grupos com crianças e adolescentes com sobrepeso $(n=15)$, obesidade $(n=30)$ e controle $(n=21)$. O perfil lipídico foi avaliado por meio do colesterol total, LDL-colesterol, HDLcolesterol e triglicerídeos. A peroxidação de lipídeos no plasma foi medida pelo marcador malonildialdeído (MDA). A análise estatística foi realizada através do teste $t$ de Student, teste de Tukey, ANOVA e correlação de Pearson.

Resultados: As alterações de colesterol total e LDL-colesterol estavam mais presentes nos grupos sobrepeso e obesidade masculinos. O HDL-colesterol mostrou-se em condições limítrofes nos grupos sobrepeso e obesidade em ambos sexos. As maiores concentrações de triglicerídeos foram registradas no grupo obesidade feminino. Identificou-se elevada peroxidação de lipídeos no plasma no grupo obesidade, principalmente no sexo masculino.

Conclusões: $\mathrm{Na}$ amostra estudada, maiores alterações do perfil lipídico foram observadas no sexo masculino nos grupos sobrepeso e obesidade, e a peroxidação de lipídeos estava mais evidente no grupo obesidade em ambos sexos.

J Pediatr (Rio J). 2004;80(1):23-8: Análise de lipídeos, peroxidação de lipídeos, obesidade.

\section{Introdução}

Evidências sugerem que a prevalência do sobrepeso e da obesidade na faixa pediátrica tem aumentado significativamente, apontando para uma epidemia mundial. Este fato é preocupante, uma vez que as alterações metabólicas e conseqüências oriundas da obesidade, antes evidentes apenas em adultos, já podem ser observadas na faixa etária mais jovem ${ }^{1}$.

1. Mestre em Ciências Farmacêuticas, Universidade Federal do Rio Grande do Norte (UFRN).

2. Mestre. Professor assistente, Departamento de Pediatria, UFRN.

3. Doutora. Professora adjunta, Departamento de Toxicologia, UFRN.

4. Professora adjunta, Departamento de Análises Clínicas, UFRN.

5. Doutora. Professora adjunta, Departamento de Nutrição, UFRN

Fonte financiadora: Fundo de Pesquisa - Pró-Reitoria de Pesquisa da UFRN/CNPq (processo 300127/91-0)

Artigo submetido em 07.11.02, aceito em 15.10.03.

\begin{abstract}
Objective: To study the plasma lipid profile and lipid peroxidation in overweight or obese children and adolescents receiving care at the pediatric endocrinology clinic in HOSPED/UFRN, a university hospital.

Methods: Three groups were studied: overweight $(n=15)$, obese $(n=30)$ and control $(n=21)$ children and adolescents. To evaluate plasma lipid profile, total cholesterol, LDL-cholesterol, HDL-cholesterol and triglyceride levels were measured. Lipid peroxidation was determined by measuring malondialdehyde concentration. Data were analyzed using Student's $t$ test, Tukey test, ANOVA and Pearson's correlation.
\end{abstract}

Results: Altered levels of total and LDL-cholesterol were observed mainly in overweight or obese males. HDL-cholesterol was borderline in the overweight and obese groups of both sexes. Obese females had the highest levels of triglycerides. Increased plasma lipid peroxidation was observed mainly in obese males.

Conclusion: In the present population, the greatest alterations in lipid profile were observed in obese and overweight males. Plasma lipid peroxidation was more evident in obese males and females.

J Pediatr (Rio J). 2004;80(1):23-8: Lipid profile, plasma lipid peroxidation, obesity.

No Brasil, as mudanças demográficas, socioeconômicas e epidemiológicas ao longo do tempo permitiram que ocorresse a denominada transição nos padrões nutricionais. Comparando-se os dados do Estudo Nacional da Despesa Familiar (ENDEF-1974/75) com os dados da Pesquisa sobre Padrões de Vida (PPV) realizada em 1996/97, verificou-se, nas regiões sudeste e nordeste, um aumento na prevalência de sobrepeso e obesidade de $4,1 \%$ para $13,9 \%$ em crianças e adolescentes de 6 a 18 anos $^{2}$.

O fator de risco potencial para a criança tornar-se obesa é a freqüência de obesidade entre os familiares, devido à soma da influência genética e dos fatores ambientais, como os hábitos alimentares, que determinam a ingestão de energia e o estilo de vida da família ${ }^{3}$.

Estudos epidemiológicos têm mostrado uma maior incidência de doenças cardiovasculares em populações 
com altos níveis de colesterol total e de LDL-colesterol e baixos níveis de HDL-colesterol ${ }^{4,5}$. Um estudo em crianças e adolescentes com diagnóstico de obesidade, com mediana de idade de 11 anos e 10 meses para os meninos e 10 anos e 9 meses para as meninas, já observou valores alterados de HDL em $68,75 \%$ dos participantes, uma elevada incidência de triglicérides (35\%) e VLDL (37\%) aumentados 6 .

A literatura é escassa em relação a estudos de prevenção envolvendo obesidade, dislipidemia e marcadores de peroxidação lipídica, principalmente em crianças e adolescentes. No entanto, quando as complicações da obesidade já estão instaladas, como nas doenças arteriais coronarianas, são observadas correlações positivas entre os marcadores de peroxidação lipídica (MDA), colesterol total e triglicérides, e correlações negativas com o $\mathrm{HDL}^{7}$.

A oxidação da LDL pode apresentar-se em alvos fosfolipídicos específicos na superfície da partícula (a chamada "LDL minimamente oxidada") e estender-se até a oxidação dos lipídeos e proteínas internas da partícula. Como conseqüência, ocorre o acúmulo de subprodutos, tais como o malonildialdeído (MDA), considerado como marcador do processo de oxidação no organismo ${ }^{8}$. Assim, a LDL oxidada, além de transformar macrófagos em células espumosas, também aumenta a adesão, ativação e migração dos monócitos, propiciando a formação das placas de ateromas ${ }^{9}$.

A detecção precoce da peroxidação lipídica em crianças e adolescentes obesas pode auxiliar na conduta clínica pediátrica quanto ao retardo e à prevenção de complicações crônicas. Com base nesses fundamentos, este estudo teve como objetivo investigar o perfil lipídico e a peroxidação de lipídeos no plasma em crianças e adolescentes com sobrepeso e obesidade.

\section{Metodologia}

Foi realizado um estudo caso-controle pareado com crianças e adolescentes de ambos os sexos, no período de janeiro de 2001 a dezembro de 2002, no Ambulatório de Endocrinologia Pediátrica do Hospital de Pediatria da UFRN, Natal, RN. Avaliou-se uma amostra com 45 pacientes entre os 63 que freqüentavam o ambulatório neste período, dos quais 15 apresentavam diagnóstico de sobrepeso, e 30, de obesidade. O tamanho amostral, incluindo os grupos sobrepeso, obesidade e controle (66), foi calculado com grau de confiança de $95 \%$ e margem de erro de $6 \%$. Foram selecionados pacientes que estavam de acordo com os critérios estabelecidos para inclusão: crianças ou adolescentes com idade entre 6 e 16 anos, diagnóstico de sobrepeso ou obesidade de acordo com o índice de massa corporal (IMC); e para exclusão: ausência de síndromes genéticas associadas à obesidade ou quaisquer doenças crônicas. Do total de 63 pacientes, não foram incluídos cinco pacientes que apresentavam idade inferior a 6 anos, quatro com doença crônica detectada ou relatada no momento da consulta, dois com síndrome de Prader-Willi e sete que não compareceram para a coleta de exames, constituindo-se a amostra final de 45 participantes.
Foi formado um grupo controle com 21 crianças e adolescentes entre os 30 selecionados no serviço de triagem do Ambulatório Geral da UFRN, submetido à avaliação clínica pelo médico e nutricionista responsável pelo estudo. Dos 30 participantes selecionados para constituir o grupo controle, foram excluídos quatro que apresentaram alteração no perfil lipídico e cinco que não compareceram para a coleta de exames. O grupo controle foi composto observando-se os seguintes critérios de inclusão: eutrofia, considerando o P50 do IMC, perfil lipídico plasmático com valores desejáveis e ausência de doenças crônicas. Na execução do pareamento dos grupos, alguns pacientes do grupo controle foram pareados com mais de um paciente, desde que apresentassem o mesmo sexo, idade (variando até 6 meses) e grau de maturação sexual (impúbere e púbere) segundo a classificação de Tanner ${ }^{10}$.

O estudo foi aprovado pelo Comitê de Ética em Pesquisa da UFRN. O consentimento esclarecido por escrito foi obtido por parte dos pais ou responsáveis após os mesmos terem recebido informações detalhadas e específicas sobre os riscos e benefícios da pesquisa.

A avaliação antropométrica foi realizada pelo IMC, utilizando-se os pontos de corte estabelecidos por Must et al. ${ }^{11}$ e recomendados pela Organização Mundial da Saúde, os quais definem sobrepeso para as medidas entre os percentis 85-95, e obesidade para as medidas acima do percentil 95

O perfil lipídico foi avaliado por meio do colesterol total, LDL-colesterol, HDL-colesterol e triglicerídeos, segundo valores de referência de Kwiterovich ${ }^{12}$. As determinações foram realizadas pelo método enzimático colorimétrico, utilizando-se reagentes LABTEST. Os valores do LDL-colesterol foram obtidos por meio da equação de Friedewald.

A concentração de produtos da oxidação de lipídeos no plasma (MDA) foi feita pela determinação do produto de reação entre o ácido tiobarbitúrico (TBA) e os aldeídos produzidos, durante a oxidação de lipídeos. Os resultados foram expressos por hmoles/mL de plasma ${ }^{13}$.

Os dados obtidos foram analisados pelo software Statistica versão 5 , com aplicação de testes $t$ student para variável IMC, análise de variância (ANOVA), teste de Tukey e correlação de Pearson para as variáveis CT, LDL, HDL, TGL e MDA entre os grupos. Foi estabelecido o nível de significância $(p<0,05)$.

\section{Resultados}

Das 66 crianças e adolescentes estudados, encontramos no grupo sobrepeso masculino uma média de idade de $11,53 \pm 2,38$ anos (variação: 8,6 - 14,2), e no grupo femi-

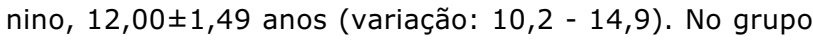
obesidade, a idade média correspondeu, no sexo masculino, a 11,05 $\pm 1,78$ anos (variação: 7,6 - 13,5), e no feminino, a $10,75 \pm 3,17$ anos (variação: 6,1 - 15,6). No grupo controle masculino, a idade média foi de $11,24 \pm 2,37$ anos (variação: $7,8-14,7)$, e no grupo controle feminino, $11,51 \pm 3,12$ anos (variação: 6,0 - 15,1). 
Em relação ao desenvolvimento puberal, 14 (21,2\%) meninos estavam no estágio $1,12(18,2 \%)$ no estágio 2 , e quatro $(6 \%)$ no estágio 3 . Quanto às meninas, $10(15,1 \%)$ estavam no estágio 1 , nove $(13,7 \%)$ no estágio 2 , oito $(12,1 \%)$ no estágio 3 , e nove $(13,6 \%)$ estavam no estágio 4 (Tabela 1).

$\mathrm{Na}$ Tabela 2, apresentamos as características gerais dos grupos estudados. O IMC diferiu de forma estatisticamente significante $(p=0,001)$ entre os grupos sobrepeso e obesidade em relação ao grupo controle.

A Tabela 3 apresenta o perfil lipídico dos participantes. Os grupos sobrepeso e obesidade masculinos apresentaram concentrações limítrofes de colesterol total. Maiores valores de LDL-colesterol, também considerados limítrofes, foram detectados no grupo sobrepeso masculino, porém sem diferença estatística significante. Os dados do HDL-colesterol nos grupos sobrepeso e obesidade em ambos os sexos situam-se nos valores de referência limítrofes. A medida de triglicerídeos do grupo obeso feminino apresentou valores elevados, com diferença estatisticamente significante $(p=0,006)$ entre o grupo obesidade feminino e o grupo controle masculino.
Pode-se observar, na Figura 1, o registro de valores maiores no MDA plasmático no grupo obeso masculino, diferentes dos controles masculinos ( $p=0,0002)$ e femininos ( $p=0,0005)$; e também no sobrepeso feminino $(p=0,0156)$. O grupo obeso feminino comparado aos controles demonstrou diferença significante somente em relação ao sexo masculino ( $p=0,0243$ ).

Observamos, no grupo obesidade masculino, uma correlação positiva $(r=0,5194, p=0,057)$ entre o IMC e o colesterol total, e, no feminino, uma correlação negativa ( $r=-0,4280, p=0,098)$ entre o IMC e o HDL-colesterol, porém sem significância estatística. Não foram identificadas correlações significantes entre as variáveis do perfil lipídico e o MDA.

\section{Discussão}

Os resultados deste estudo estão em concordância com um estudo piloto previamente realizado em um subgrupo do mesmo Ambulatório, no qual registrou-se a prevalência de $76,9 \%$ de crianças e adolescentes com obesidade e $23,1 \%$ com sobrepeso ${ }^{14}$.

Tabela 1 - Classificação do estadiamento puberal das crianças e adolescentes dos grupos sobrepeso, obesidade e controle atendidos no Ambulatório de Endocrinologia Pediátrica da UFRN

\begin{tabular}{|c|c|c|c|c|c|c|c|c|c|c|}
\hline \multicolumn{2}{|c|}{ Grupos } & \multicolumn{8}{|c|}{ Estágio puberal (Tanner) } & \multirow{3}{*}{$\begin{array}{c}\text { Total } \\
10\end{array}$} \\
\hline \multirow{2}{*}{$\begin{array}{l}\text { Diagnóstico } \\
\text { Controle }\end{array}$} & \multirow{2}{*}{$\begin{array}{l}\text { Sexo } \\
\text { Masculino }\end{array}$} & \multicolumn{2}{|c|}{ M1(G1)P1 } & \multicolumn{2}{|c|}{ M2(G2)P2 } & \multicolumn{2}{|c|}{ M3(G3)P3 } & \multicolumn{2}{|c|}{ M4(G4)P4 } & \\
\hline & & 5 & $7,6 \%$ & 4 & $6,1 \%$ & 1 & $1,5 \%$ & 0 & $0,0 \%$ & \\
\hline Controle & Feminino & 3 & $4,5 \%$ & 4 & $6,1 \%$ & 1 & $1,5 \%$ & 3 & $4,5 \%$ & 11 \\
\hline Obesidade & Masculino & 8 & $12,1 \%$ & 5 & $7,6 \%$ & 1 & $1,5 \%$ & 0 & $0,0 \%$ & 14 \\
\hline Obesidade & Feminino & 7 & $10,6 \%$ & 1 & $1,5 \%$ & 4 & $6,1 \%$ & 4 & $6,1 \%$ & 16 \\
\hline Sobrepeso & Masculino & 1 & $1,5 \%$ & 3 & $4,5 \%$ & 2 & $3,0 \%$ & 0 & $0,0 \%$ & 6 \\
\hline Sobrepeso & Feminino & 0 & $0,0 \%$ & 4 & $6,1 \%$ & 3 & $4,5 \%$ & 2 & $3,0 \%$ & 9 \\
\hline Total & & 24 & $36,3 \%$ & 21 & $31,9 \%$ & 12 & $18,1 \%$ & 9 & $13,6 \%$ & 66 \\
\hline
\end{tabular}

Tabela 2 - Características gerais das crianças e adolescentes dos grupos sobrepeso, obesidade e controle atendidos no Ambulatório de Endocrinologia Pediátrica da UFRN

\begin{tabular}{|c|c|c|c|c|c|c|}
\hline \multirow[b]{2}{*}{$\begin{array}{l}\text { Características } \\
\text { gerais }\end{array}$} & \multicolumn{2}{|c|}{ Sobrepeso } & \multicolumn{2}{|c|}{ Obesidade } & \multicolumn{2}{|c|}{ Controle } \\
\hline & $\begin{array}{c}\text { Masculino } \\
(n=6)\end{array}$ & $\begin{array}{c}\text { Feminino } \\
(n=9)\end{array}$ & $\begin{array}{c}\text { Masculino } \\
(n=14)\end{array}$ & $\begin{array}{c}\text { Feminino } \\
(n=16)\end{array}$ & $\begin{array}{c}\text { Masculino } \\
(n=10)\end{array}$ & $\begin{array}{c}\text { Feminino } \\
(n=11)\end{array}$ \\
\hline Idade (anos) & $11,53 \pm 2,38$ & $12,00 \pm 1,49$ & $11,05 \pm 1,78$ & $10,75 \pm 3,17$ & $11,24 \pm 2,37$ & $11,51 \pm 3,12$ \\
\hline Altura (cm) & $145,50 \pm 14,79$ & $148,09 \pm 7,33$ & $148,78 \pm 11,02$ & $146,29 \pm 15,18$ & $149,27 \pm 15,87$ & $141,15 \pm 18,38$ \\
\hline Peso (kg) & $53,23 \pm 16,02$ & $48,82 \pm 6,50$ & $61,00 \pm 17,52$ & $60,69 \pm 20,34$ & $40,30 \pm 12,61$ & $35,95 \pm 11,52$ \\
\hline IMC $\left(\mathrm{kg} / \mathrm{m}^{2}\right)$ & $24,68 \pm 3,91^{*}$ & $22,22 \pm 1,13^{*}$ & $26,45 \pm 3,37^{*}$ & $27,45 \pm 4,29 *$ & $17,61 \pm 1,79$ & $17,47 \pm 1,76$ \\
\hline
\end{tabular}

Média \pm desvio padrão.

* Houve diferença significante (ANOVA) do IMC entre os grupos sobrepeso e obesidade quando comparados com o controle $(p=0,001)$. $\mathrm{IMC}=$ peso atual/altura $\left(\mathrm{m}^{2}\right)$. 
Tabela 3 - Perfil lipídico das crianças e adolescentes dos grupos sobrepeso, obesidade e controle atendidos no Ambulatório de Endocrinologia Pediátrica da UFRN

\begin{tabular}{|c|c|c|c|c|c|c|}
\hline \multirow[b]{2}{*}{ Perfil lipídico } & \multicolumn{2}{|c|}{ Sobrepeso } & \multicolumn{2}{|c|}{ Obesidade } & \multicolumn{2}{|c|}{ Controle } \\
\hline & $\begin{array}{l}\text { Masculino } \\
(n=6)\end{array}$ & $\begin{array}{c}\text { Feminino } \\
(n=9)\end{array}$ & $\begin{array}{l}\text { Masculino } \\
(n=14)\end{array}$ & $\begin{array}{c}\text { Feminino } \\
(n=16)\end{array}$ & $\begin{array}{l}\text { Masculino } \\
(n=10)\end{array}$ & $\begin{array}{c}\text { Feminino } \\
(n=11)\end{array}$ \\
\hline $\begin{array}{l}\text { Colesterol total } \\
(\mathrm{mg} / \mathrm{dl})\end{array}$ & $182,42 \pm 43,68$ & $152,78 \pm 39,10$ & $173,82 \pm 41,24$ & $148,97 \pm 43,68$ & $153,15 \pm 22,38$ & $151,07 \pm 23,32$ \\
\hline $\begin{array}{l}\text { LDL-colesterol } \\
(\mathrm{mg} / \mathrm{dl})\end{array}$ & $125,62 \pm 43,04$ & $93,24 \pm 40,48$ & $109,56 \pm 39,08$ & $82,17 \pm 39,37$ & $92,05 \pm 26,78$ & $88,03 \pm 18,60$ \\
\hline $\begin{array}{l}\text { HDL-colesterol } \\
(\mathrm{mg} / \mathrm{dl})\end{array}$ & $37,92 \pm 8,95$ & $39,72 \pm 8,62$ & $40,18 \pm 10,29$ & $39,03 \pm 9,17$ & $50,00 \pm 18,33$ & $46,46 \pm 13,46$ \\
\hline $\begin{array}{l}\text { Triglicerídeos } \\
(\mathrm{mg} / \mathrm{dl})\end{array}$ & $94,75 \pm 44,05$ & $99,83 \pm 65,02$ & $120,43 \pm 48,81$ & $139,05 \pm 68,43 *$ & $55,31 \pm 16,27$ & $82,58 \pm 25,38$ \\
\hline
\end{tabular}

Média \pm desvio padrão.

* Existe diferença significativa (ANOVA) para os triglicerídeos entre o grupo obeso feminino e o grupo controle masculino ( $p=0,006)$.

Verificamos que a variável IMC não apresentou distinção entre sexo nos grupos sobrepeso e obesidade; o mesmo foi observado por Valverde et al. ${ }^{6}$ em crianças e adolescentes.

O perfil lipídico dos grupos sobrepeso e obesidade revelaram concentrações médias variando nos intervalos limítrofes ou indesejáveis, sobretudo no sexo masculino. Os valores de colesterol total dos grupos sobrepeso e obesidade estavam semelhantes aos constatados na população americana, cujas concentrações de colesterol nos meninos foram de $168 \mathrm{mg} / \mathrm{dl}$, e nas meninas, 173 $\mathrm{mg} / \mathrm{dl}^{4}$; além disso, os valores estavam maiores do que os obtidos por Moura et al. ${ }^{15}$.
Nos grupos sobrepeso e obesidade, houve uma maior tendência à hipercolesterolemia, preferencialmente no sexo masculino quando comparado ao feminino, o que pode ter sido influenciado pela freqüência de meninas púberes (nove no grupo sobrepeso e nove no obesidade). As alterações hormonais presentes neste estágio de vida podem atuar como um fator de proteção contra alterações do perfil lipídico. Antes e após a menarca, as mudanças no perfil lipídico são sensíveis à influência dos hormônios sexuais, principalmente o estrógeno, que exerce um efeito favorável sobre as lipoproteínas, aumentando os níveis de HDL-c e diminuindo o LDL-c. Neste contexto, o sexo feminino é beneficiado durante a adolescência e a vida adulta ${ }^{16}$.

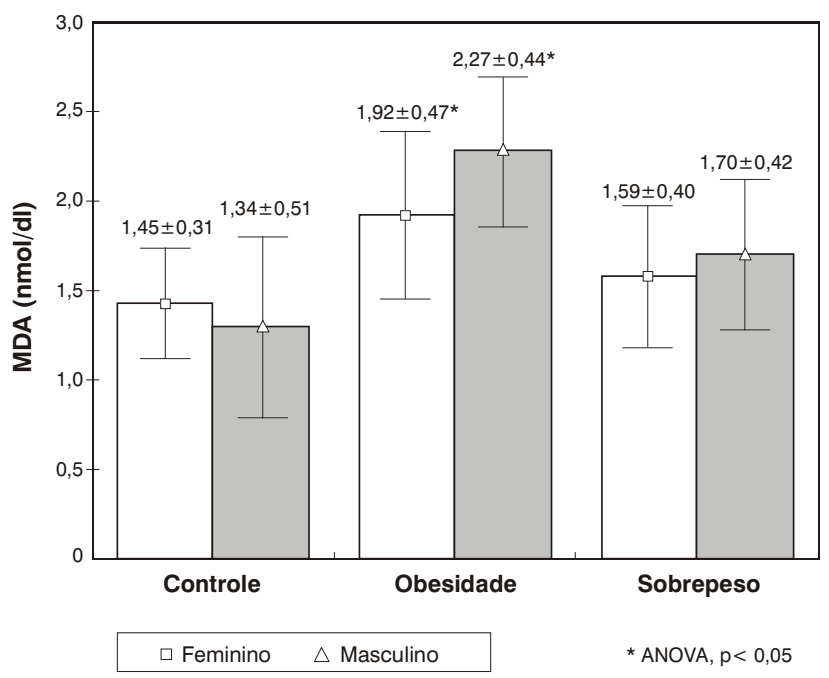

Figura 1 - MDA plasmático das crianças e adolescentes dos grupos sobrepeso, obesidade e controle atendidos no Ambulatório de Endocrinologia Pediátrica da UFRN 
Observações contrárias foram registradas em escolares de Campinas ${ }^{17}$.

O estudo de Framingham indicou uma contínua elevação de risco para doença arterial coronariana à medida que o colesterol ultrapassa o nível de $180 \mathrm{mg} \%{ }^{18}$. Analisando os grupos sobrepeso e obesidade, detectamos 12 crianças e adolescentes nessa situação de risco, quatro do sexo feminino e oito do sexo masculino.

Os valores de LDL-colesterol do nosso estudo foram superiores aos de Moura et al. ${ }^{15}$, o que pode ser atribuído ao elevado consumo de gorduras saturadas e à baixa ingestão de fibras dietéticas. Salientando que, no grupo sobrepeso masculino, $50 \%$ da amostra estava com valores indesejáveis para a fração LDL-colesterol, torna-se indispensável, nos serviços de saúde, a conduta de busca seletiva de crianças e adolescentes com risco de sobrepeso ou quaisquer outros fatores de risco evidentes para a progressão do processo aterosclerótico.

O HDL-colesterol, que atua como fator de proteção contra as doenças cardíacas, foi caracterizado em condições limítrofes nos nossos grupos de pacientes, com valores abaixo dos observados por Webber et al. ${ }^{4} \mathrm{e}$ discretamente maiores que os de Moura \& Sonati ${ }^{19}$. Nos grupos sobrepeso e obesidade, detectamos que 10 meninas e 12 meninos apresentavam HDL $<40 \mathrm{mg} / \mathrm{dl}$. Gerber \& Zielinsky, analisando os fatores de risco para aterosclerose na infância, identificaram que 31 das crianças apresentavam $\mathrm{HDL}<40 \mathrm{mg} / \mathrm{dl}$ associado a colesterol total $>180 \mathrm{mg} / \mathrm{dl}^{20}$. Verificamos que quatro $(8,7 \%)$ participantes dos grupos sobrepeso e obesidade se encontravam neste limiar de risco aterogênico.

Constatamos uma maior prevalência de valores indesejáveis de TGL nos grupos obesidade masculino $(35,7 \%)$ e feminino $(50,0 \%)$, corroborando os achados de Valverde et al. 5 , que, investigando 74 crianças e adolescentes obesos, obtiveram valores alterados de HDL em $68,75 \%$ da amostra, além de valores elevados de TL e VLDL.

Alterações de MDA como marcador de peroxidação lipídica observadas em crianças e adolescentes com sobrepeso ou obesidade podem ser considerados como um fator de risco potencial para complicações cardiovasculares. Altos índices de peroxidação lipídica têm sido identificados no soro de pacientes com angina pectoris ${ }^{21}$ e doenças arteriais coronarianas ${ }^{22}$.

A peroxidação lipídica no plasma foi constatada como uma alteração marcante da obesidade, devido aos maiores valores de MDA obtidos, sobretudo nos obesos do sexo masculino, os quais também apresentavam concentrações limítrofes de colesterol total. A análise dos intervalos de variação desta variável demonstra que a menor concentração encontrada no grupo obesidade $(1,92 \pm 0,47)$ é superior ao valor médio dos controles $(1,34 \pm 0,51)$. Observaram-se também, no grupo sobrepeso masculino (que apresentou o maior percentual de valores indesejáveis para o LDL-c, $50 \%$ ), concentrações elevadas de MDA, embora sem demonstrar diferença estatisticamente significante.

Conclui-se que, na amostra estudada, houve distinção entre os sexos em relação às alterações de perfil lipídico, com elevadas concentrações de colesterol total e LDLcolesterol no sexo masculino nos grupos sobrepeso e obesidade, enquanto que as de triglicerídeos predominaram no sexo feminino no grupo obesidade. A elevada peroxidação lipídica no plasma detectada no grupo obesidade em ambos sexos constitui uma alerta para a conduta clínica da obesidade em faixa pediátrica, devendo ser considerada em atenção às possíveis complicações crônicas no curso da doença.

\section{Agradecimentos}

À nutricionista Ana Sueli de Andrade pelo auxílio em diversas atividades deste estudo e às crianças e adolescentes participantes.

\section{Referências}

1. Styne DM. Childhood and adolescent obesity. Prevalence and significance. Pediatr Clin North Am. 2001;48:823-53.

2. Wang $Y$, Monteiro C, Popkin BM. Trends of obesity and underweight in older children and adolescents in the United States, Brazil, China, and Russia. Am J Clin Nutr. 2002; 75:971-7.

3. Nguyen VT, Larson DE, Johnson RK, Goran MI. Fat intake and adiposity in children of lean and obese parents. Am J Clin Nutr. 1996;63:507-13.

4. Webber LS, Organian V, Luepker RV, Feldman HA, Stone EJ, Elder JP, et al. Cardiovascular risk factors among third grade children in four regions of the United States. Am J Epidemiol. 1995; 141:428-39.

5. Freedman DS, Dietz WH, Srinivasan SR, Berenson GS. The Relation of overweight to cardiovascular risk factors among children and adolescents: The Bogalusa Heart Study. Pediatrics. 1999; 103:1175-82.

6. Valverde $M A$, Vítolo $M R$, Patin RV, Escrivão MAMS, Oliveira FLCO, Ancona-Lopez F. Investigação de alterações no perfil lipídico de crianças e adolescentes obesos. Arch Latinoam Nutr. 1999;49:338-43.

7. Mahapatra S, Padhiary K, Mishra TK, Nayak N, Satpathy M. Study on body mass index, lipid profile and lipid peroxidation status in coronary artery disease. J Indian Med Assoc. 1998;96:39-41.

8. Navab M, Berliner JA, Watson AD. The Yin and Yang of oxidation in the development of the fatty streak. A review based on the 1994 George Lyman Duff Memorial Lecture. Arterioscler Thromb Vasc Biol. 1996;16:831-42.

9. Ross R. The pathogenesis of atherosclerosis: a perspective for the 1990s. Nature. 1993;362:801-09.

10. Tanner JM, Whitehouse RH. Clinical longitudinal standards for height, weight, height velocity, weight velocity, and stages of puberty. Arch Dis Child. 1976;62:57-62.

11. Must A, Dallal GE, Dietz WH. Reference data for obesity: $85^{\text {th }}$ and $95^{\text {th }}$ percentiles of body mass index (wt/ht2) - a correction. Am J Clin Nutr. 1991;53(4):839-46.

12. Kwiterovich POJR. Beyond Cholesterol: The Johns Hopkins Complete guide for Avoiding Heart Disease. Baltimore: The John Hopkins Press; 1989.

13. Yagi K. Lipid peroxides in biology and medicine. Washington: Acad Press; 1984.

14. Lima SCVC, Arrais RF. Estudo do perfil lipídico e hábitos alimentares das crianças portadoras de sobrepeso e obesidade atendidas no ambulatório de Endocrinologia Pediátrica da UFRN. Rev Saúde. 1999;13:51-5.

15. Moura EC, Nakamura SY, Romero VSV. Níveis de colesterol em escolares da primeira série de uma escola pública de Campinas, São Paulo, Brasil. Rev Nutr PUCCAMP. 1997;10:63-9.

16. Rabelo LM. Fatores de risco para doença aterosclerótica na adolescência. J Pediatr (Rio J). 2001;77:153-64. 
17. Moura EC, Castro CM, Mellin AS, Fiqueiredo DB. Perfil lipídico em escolares de Campinas, SP, Brasil. Rev Saúde Pública. 2000; 34:499-05.

18. Castelli WP. Epidemiology of coronary heart disease: The Framinghan Study. Am J Med. 1984;76(2A):4-12.

19. Moura EC, Sonati JG. Perfil lipídico de dietas e sua relação com os níveis de colesterolemia em escolares de uma escola pública de Campinas, São Paulo, Brasil. Rev Nutr PUCCAMP. 1998; 11:69-5.

20. Gerber ZRS, Zielinsky P. Fatores de risco de aterosclerose na infância. Um estudo epidemiológico. Arq Bras Cardiol. 1997; 69:231-6.

21. Mendis S, Sobotka PA, Leja FL, Euler DE. Breath pentane and plasma lipid peroxides in ischemic heart disease. Free Radic Biol Med. 1995;19:679-84.
22. Sakuma $N$, Hibino $T$, Sato $T$, Ohte $N$, Akita $S$, Tamai $N$, et al. Levels of thiobarbituric acid-reactive substance in plasma from coronary artery disease patients. Clin Biochem. 1997;30:505-07.

Correspondência:

Severina Carla Vieira Cunha Lima

Rua Epitácio Andrade, 1420/104

CEP 59022-405 - Natal, RN

Tel.: (84) 213.0095/982.8490

E-mail: scarla@samnet.com.br 